\title{
Editorial
}

\section{Enseñanzas de los inhibidores de COX-2 en el proceso de evaluación de medicamentos}

En los últimos años los fármacos inhibidores selectivos de la ciclooxigenasa 2 (COX-2), o coxib, han dado lugar a un revuelo sin apenas precedentes. Los coxib son un tipo de antiinflamatorios no esteroideos (AINES) que se desarrollaron siguiendo la hipótesis de que la COX-2 era la fuente de las prostraglandinas E2 e I2, mediadoras de la inflamación, mientras que la ciclooxigenasa 1 (COX-1) era la fuente de las mismas prostraglandinas en el epitelio gástrico. Los coxib tendrían, por tanto, menores efectos adversos gastrointestinales que los antiinflamatorios no esteroideos clásicos. Sin embargo, la historia de su desarrollo clínico es poco edificante. Los dos ensayos decisivos, cada uno con más de 8000 participantes, se publicaron en 2000, el CLASS (Celecoxib Long-term Arthritis Study, comparación de celecoxib con diclofenaco o ibuprofeno con respecto a las complicaciones de úlcera gastrointestinal en pacientes con artrosis o artritis reumatoide) ${ }^{1}$ y el VIGOR (Vioxx Gastrointestinal Outcomes Research, similar comparación de rofecoxib con naproxeno en pacientes con artritis reumatoide $)^{2}$. La lectura del CLASS mostraba un optimismo no sustentado en los datos, pues el celecoxib no reducía significativamente la incidencia de complicaciones ulcerosas. Cuando poco después se divulgó la información recopilada por la Food and Drug Administration (FDA) se supo que el ensayo CLASS era la agregación de dos protocolos distintos, uno para diclofenaco, de 12 meses de duración, y otro para ibuprofeno, de 16 meses, que deberían haberse analizado separadamente; que efectuaron análisis de subgrupos no autorizados en el protocolo original; y, lo más grave, que el análisis se limitó a 6 meses de seguimiento, cuando el tiempo previsto era de 12 meses y para entonces la toxicidad del celecoxib era igual a la de los AINES clásicos ${ }^{3-5}$. Esta información se ocultó al director de la revista donde se publicó. En el VIGOR, rofecoxib redujo a la mitad la toxicidad gastrointestinal del naproxeno pero multiplicó por cuatro la frecuencia de infarto agudo de miocardio. En la publicación hubo omisiones importantes en la descripción de datos contrarios al rofecoxib, como que el número de efectos adversos graves era significativamente superior en el grupo de rofecoxib que en el de naproxeno (riesgo relativo 1,21 -intervalo de confianza 95\%: 1,04-1,40), como figuraba en el informe de la FDA publicado poco antes ${ }^{6,7}$. Al revisar los datos de estos ensayos y de otros dos previos no publicados, autores independientes apreciaron un riesgo mayor de acontecimientos cardiovasculares con el uso de coxib y propusieron un ensayo controlado aleatorizado para esclarecerlo ${ }^{8}$.

En España, se produjo un desgraciado incidente al hilo de estos acontecimientos: la compañía fabricante de rofecoxib, Merck Sharp \& Dohme, demandó a la revista 'Butlletí Groc', dirigida por Josep-Ramon Laporte, para exigir una rectificación de un artículo de julio-septiembre de 2002 que se hacía eco de estas irregularidades $^{9}$. Al final, al ser desestimada la demanda, quedó avalada la independencia en la información sobre medicamentos.

La sospecha de una toxicidad cardiovascular mayor no se abordó explícitamente mientras las compañías gastaban enormes sumas para hacer propaganda de sus productos. En Estados Unidos cada mes había más de 10 millones de prescripciones de rofecoxib ${ }^{7}$ y las ventas mundiales de los coxib se han estimado en unos 10.000 millones de dólares ${ }^{10}$. Sin embargo, en septiembre de 2004 Merck decidió retirar rofecoxib del mercado al encontrar en el ensayo APPROVe, para la prevención de pólipos neoplásicos de colon recurrentes, que, en comparación con placebo, este medicamento casi duplicaba la tasa de acontecimientos trombóticos cardiovasculares (sobre todo infarto agudo de miocardio e ictus trombótico) ${ }^{11}$. Un metanálisis acumulado indicó que ya en 2000 había evidencia suficiente de esa toxicidad cardiovascular ${ }^{12}$. Al mismo tiempo, también se suspendió otro ensayo de prevención de adenomas colorrectales por aumento de complicaciones cardiovasculares (Ade- 
noma Prevention with Celecoxib -APC-, de financiación pública y privada), con celecoxib ${ }^{13}$, que no había mostrado toxicidad cardiovascular en el ensayo CLASS ${ }^{1}$; y se publicaron los resultados de un ensayo que encontraba que el tratamiento con parecoxib y valdecoxib casi cuadriplicaba el riesgo de acontecimientos cardiovasculares en pacientes sometidos a cirugía cardiaca ${ }^{14}$, que fue el único ensayo diseñado expresamente para evaluar los efectos adversos no gastrointestinales de inhibidores de COX-2. Todo esto sugiere una toxicidad cardiovascular común a todos los coxib. Posteriormente, la FDA, la Agencia Europea del Medicamento (EMEA) y otras agencias tomaron medidas para restringir su uso ${ }^{15,16}$.

Toda esta historia de los coxib se suma a casos recientes de los inhibidores de la recaptación de la serotonina causantes de suicidio juvenil y la cerivastatina causante de rabdomiolisis, en donde el fabricante ocultó información relevante ${ }^{17}$. Las irregularidades de los coxib van desde el diseño, el análisis y la publicación de los ensayos hasta el proceso de farmacovigilancia post-autorización y el papel de las autoridades reguladoras, tanto la FDA como la EMEA $^{18}$. Irregularidades de consecuencias incalculables, ante todo un aumento indeseado potencial de morbilidad y mortalidad, y la quiebra en la confianza que la sociedad deposita en las instituciones de salud pública que regulan el proceso de autorización de medicamentos.

Múltiples propuestas se pueden apuntar para evitar la repetición de estos hechos en el futuro: el registro obligatorio de todos los ensayos clínicos desde su inicio tanto para favorecer su divulgación como para garantizar la congruencia del análisis con el protocolo previsto ${ }^{19,20}$; la necesidad de independencia financiera entre los investigadores de ensayos clínicos y revisiones sistemáticas y la industria farmacéutica ${ }^{19,21}$; prestar mayor atención a la evaluación de los efectos adversos en el diseño y análisis de los ensayos clínicos, a los que se concede menor importancia que a la evaluación de eficacia ${ }^{22}$, con la paradoja de que rofecoxib fue aprobado con datos de seguridad de 5000 pacientes y se retiró del mercado cuando ya lo podían haber tomado 80 millones de personas; exigencia de divulgación automática de los efectos adversos de los medicamentos desde el término de la investigación para su autorización ${ }^{19}$; transformar por entero la farmacovigilancia de los efectos adversos de los medicamentos para hacerla efectiva ${ }^{17,23}$; la necesidad de separar los organismos de evaluación de la seguridad de los medicamentos de aquellos encargados de su autorización ${ }^{24}$, que ha conducido al gobierno de Estados Unidos a crear una agencia independiente de la FDA, la Drug Safety Oversight Board, paso que Europa todavía no ha dado; activar el papel de las autoridades reguladoras, que pueden haber hecho dejación de su responsabilidad en este caso al adoptar un papel pasivo; garantizar la independencia completa de las autoridades reguladoras de la industria farmacéutica, situación difícil de asegurar en el momento actual, en que la FDA y la EMEA se financian en buena parte por la industria; favorecer la investigación en medicamentos independiente de la industria que, lamentablemente, la reciente directiva sobre ensayos clínicos de la Unión Europea dificulta, y proveer de recursos económicos públicos para ello; y exigir el cumplimiento de un código de conductas éticas por la industria farmacéutica relativo a la transparencia de sus datos y a la diligencia en el diseño de estudios sobre efectos adversos.

El sistema actual de evaluación de nuevos medicamentos debe someterse a cambios profundos en interés de todos los protagonistas implicados, pacientes, clínicos, investigadores, autoridades y fabricantes. Alarmas de toxicidad semejantes no se deberían repetir.

Fernando García López

Unidad de Epidemiología Clínica Hospital Universitario Puerta de Hierro

Madrid 


\section{Bibliografía}

1. Silverstein FE, Faich G, Goldstein JL et al. Gastrointestinal toxicity with celecoxib vs nonsteroidal anti-inflammatory drugs for osteoarthritis and rheumatoid arthritis: the CLASS study: A randomized controlled trial. Celecoxib Long-term Arthritis Safety Study. JAMA 2000; 284:1247-1255.

2. Bombardier C, Laine L, Reicin A et al. Comparison of upper gastrointestinal toxicity of rofecoxib and naproxen in patients with rheumatoid arthritis. VIGOR Study Group. N Engl J Med 2000; 343:1520-1528.

3. Hrachovec JB, Mora M. Reporting of 6-month vs 12-month data in a clinical trial of celecoxib. JAMA 2001; 286:2398-2400.

4. Wright JM, Perry TL, Bassett KL, Chambers GK. Reporting of 6-month vs 12-month data in a clinical trial of celecoxib. JAMA 2001; 286:2398-2400.

5. Jüni P, Rutjes AW, Dieppe PA. Are selective COX 2 inhibitors superior to traditional non steroidal anti-inflammatory drugs? BMJ 2002; 324:1287-1288.

6. Villalba ML, Li Q. Statistical Reviewer Briefing Document for the Advisory Committee. The Food and Drug Administration. www.fda.gov/ohrms/dockets/ac/01/briefing/3677b2_04_stats.pdf. Visualizado el 25-mayo-2005.

7. Topol EJ. Failing the public health—rofecoxib, Merck, and the FDA. N Engl J Med 2004; 351:1707-1709.

8. Mukherjee D, Nissen SE, Topol EJ. Risk of cardiovascular events associated with selective COX-2 inhibitors. JAMA 2001; 286:954-959.

9. Las supuestas ventajas de celecoxib y rofecoxib: fraude científico. Butlletí Groc 2002; 15:13-14.

10. Fitzgerald GA. Coxibs and cardiovascular disease. N Engl J Med 2004; 351:1709-1711.

11. Bresalier RS, Sandler RS, Quan H et al. Cardiovascular events associated with rofecoxib in a colorectal adenoma chemoprevention trial. N Engl J Med 2005; 352:1092-1102.

12. Jüni P, Nartey L, Reichenbach S, Sterchi R, Dieppe PA, Egger M. Risk of cardiovascular events and rofecoxib: cumulative metaanalysis. Lancet 2004; 364:2021-2029.

13. Solomon SD, McMurray JJ, Pfeffer MA et al. Cardiovascular risk associated with celecoxib in a clinical trial for colorectal adenoma prevention. N Engl J Med 2005; 352:1071-1080.

14. Nussmeier NA, Whelton AA, Brown MT et al. Complications of the COX-2 inhibitors parecoxib and valdecoxib after cardiac surgery. N Engl J Med 2005; 352:1081-1091.

15. Lenzer J. FDA advisers warn: COX 2 inhibitors increase risk of heart attack and stroke. BMJ 2005; 330:440.

16. Lenzer J. Pfizer is asked to suspend sales of painkiller. BMJ 2005; 330:862.

17. Fontanarosa PB, Rennie D, DeAngelis CD. Postmarketing surveillance—lack of vigilance, lack of trust. JAMA 2004; 292:26472650.

18. Garattini S, Bertele V. Discontinuation of Vioxx. Lancet 2005; 365:24.

19. Dieppe PA, Ebrahim S, Martin RM, Juni P. Lessons from the withdrawal of rofecoxib. BMJ 2004; 329:867-868.

20. Krleža-Jeric’ K, Chan AW, Dickersin K, Sim I, Grimshaw J, Gluud C. Principles for international registration of protocol information and results from human trials of health related interventions: Ottawa statement (part 1). BMJ 2005; 330:956-958.

21. Davidoff F, DeAngelis CD, Drazen JM et al. Sponsorship, authorship, and accountability. JAMA 2001; 286:1232-1234.

22. Vioxx: lessons for Health Canada and the FDA. CMAJ 2005; 172:5.

23. Laupacis A, Paterson JM, Mamdani M, Rostom A, Anderson GM. Gaps in the evaluation and monitoring of new pharmaceuticals: proposal for a different approach. CMAJ 2003; 169:1167-1170.

24. Horton R. Vioxx, the implosion of Merck, and aftershocks at the FDA. Lancet 2004; 364:1995-1996. 